\title{
Braulio de Zaragoza, el rey Chindasvinto y Eugenio de Toledo: imagen y opinión en el Epistularium de Braulio de Zaragoza
}

\author{
Ruth Miguel Franco
}

Universitat de les Illes Balears

ruth.miguel@uib.es

\section{Braulio of Saragossa, king Chindasvinthus and Eugenius III of Toledo: imagery and opinion in the Epistularium of Braulio of Saragossa}

Tradicionalmente se ha supuesto que las piezas que componen el Epistularium de Braulio de Zaragoza (†651) no están organizadas siguiendo ningún patrón concreto. Como prueba de esta afirmación se ha aducido el hecho de que la correspondencia entre Braulio y Eugenio III de Toledo, que se fecha cuando este último desempeñaba ya el cargo de obispo de la sede toledana, anteceda a la que Braulio intercambió con el rey Chindasvinto antes de la consagración episcopal de Eugenio, que provocó un enfrentamiento entre el obispo de Zaragoza y el monarca. Este trabajo parte del análisis global del orden del Epistularium de Braulio, que demuestra que está organizado según una estructura altamente elaborada. Dentro de esta compleja disposición, las epístolas de Braulio, Eugenio y Chindasvinto adquieren nuevos significados derivados de su lectura anticronológica y de su relación con las otras piezas de su entorno. En conclusión, se intentará demostrar que, mediante la cuidadosa organización de las epístolas y otros mecanismos formales y literarios, Braulio proyecta una imagen determinada del poder real y expresa su opinión sobre su relación con el rey Chindasvinto.

Palabras clave: Braulio de Zaragoza; Epistularium; rey Chindasvinto; Eugenio II de Toledo; clero visigodo; monarquía visigoda.
The organization of letters in the Epistularium of Braulio of Saragossa (†651) has thus far been thought not to follow any sort of logical pattern, as anti-timewise order of some epistles seems to confirm, namely, the fact that the letters between Braulio and Eugenius III of Toledo, written when Eugenius was already bishop of the Toledo see, are set before those epistles in which Braulio quarrels whith king Chindasvinthus about Eugenius' episcopal consecration. The point of departure of this work is the thorough analysis of the order of Braulio's Epistularium, which shows that this epistolary collection was organized according to a highly elaborated pattern. Within this complex structure, the epistles of Braulio, Eugenius and Chindasvinthus attain new meanings coming from anti-timewise setup and contact with surrounding epistles. In sum, we will try to show that, through careful organization and formal and literary devices, Braulio gives a precise and meaningful image of royal power and expresses his opinion about his relationships with king Chindasvinthus.

Keywords: Braulio of Saragosse; Epistularium; king Chindasvinthus; Eugenius II of Toledo; Visigothic priesthood; Visigothic monarchy. 


\section{INTRODUCCIÓN}

El Epistularium de Braulio de Zaragoza $(\uparrow 651)^{1}$ es uno de los documentos más importantes para el conocimiento de la historia de la Hispania del siglo VII, especialmente en lo que respecta a las relaciones entre el clero y la monarquía. En esta colección epistolar se nos ha conservado la correspondencia de los reyes Chindasvinto (642-653) y Recesvinto $\left(649-672^{2}\right)$ con Braulio sobre asuntos de gran relevancia histórica, como la corrección de un códice que puede ser identificado con el Fuero Juzgo (Miguel Franco 2006), o la petición que el obispo de Cesaragusta y otros personajes, entre ellos el obispo Eutropio y el $d u x$ Celso, dirigen a Chindasvinto para que asocie al trono a su hijo Recesvinto ${ }^{3}$. Además de personajes históricos, entre los corresponsales de Braulio se cuenta lo más florido de la erudición de la época: Isidoro de Sevilla, Tajón, que sería más tarde obispo en la misma sede que ocupó Braulio (García Moreno 1974, núm. 592) o el abad Fructuoso (García Moreno 1974, núm. 383).

Dentro de este conjunto epistolar son especialmente interesantes misivas como las que entre el 646 y el 647 se enviaron, por una parte, Braulio de Zaragoza y el rey Chindasvinto (642-653) y, por otra, Braulio y Eugenio (†657), que había sido arcediano en Zaragoza y discípulo de Braulio, después de su ordenación episcopal como Eugenio II de Toledo en 646 (García Moreno 1974, núm. 248; Alberto 2005, p. 13). En las ediciones las primeras cartas reciben los números 31,32 y 33 , y las últimas, las que se escribieron Braulio y Eugenio, los números 35 y $36^{4}$.

* Este trabajo se inscribe en los siguientes proyectos de investigación: FFI2009-10877, SA 120A08.

${ }^{1}$ Dentro de la bibliografía sobre la vida y obra de Braulio de Zaragoza, la aproximación más reciente es la de Martín 2006, pp. 15-91. V. también los trabajos de Valcárcel 1991, pp. 333-340, Lynch 1950.

2 Recesvinto (649-672) contó siempre los años de su reinado a partir de su asociación al trono el 20 de enero de 649 y no a partir de la muerte de su padre, Chindasvinto, en septiembre de 653, cuando pasó a ser el único rey.

3 La epístola que alrededor del 649 enviaron Braulio, el obispo Eutropio (García Moreno 1974, núm. 646) y el $d u x$ Celso (García Moreno 1974, núm. 32), recibe el número 37 en las ediciones. Sobre el reinado conjunto de Chindasvinto y Recesvinto, v. Collins 2004, pp. 82-84.

${ }^{4}$ Citaremos las epístolas de Braulio según la edición de Riesco 1975, respetando su ortografía que sigue la del codex unicus del Epistularium. Por su parte, las epístolas 35 y 36 serán citadas según Alberto 2005, pp. 399-403, y las epístolas intercambiadas con Isidoro 
El único testimonio completo del Epistularium brauliano es el manuscrito León, Archivo Capitular, 22, siglo IX ${ }^{5}$, que contiene las cartas intercambiadas entre Isidoro de Sevilla y Braulio entre los folios $38 \mathrm{v}-44 \mathrm{r}$ y el resto del epistolario en los folios $51 \mathrm{r}-88 \mathrm{v}$; en este ejemplar, las epístolas 31-36 presentan un problema, ya que están dispuestas en el orden contrario al esperable, es decir, encontramos en primer lugar las epístolas 35 y 36, fechadas en el 646/647, poco después de la ordenación episcopal de Eugenio, y, a continuación, 31, 32 y 33, que remontan al periodo inmediatamente anterior a esta consagración como obispo y que tradicionalmente se han fechado entre el 645 y el 646 (Madoz 1945, pp. 51-52; Lynch 1950, pp. 80-81).

Esta ruptura de la sucesión cronológica de las piezas ha sido aducida tradicionalmente como prueba de la falta de ordenación lógica del Epistularium. Ya la primera vez que estas epístolas salieron a la luz, su editor, Manuel Risco (1775, pp. 363-373), decidió reorganizarlas en orden cronológico, obviando la disposición en la que se encuentran en el manuscrito de León. La numeración con la que se conocen y que aquí utilizaremos remonta a esta primera edición, cuyos criterios en lo que al orden de las piezas se refiere no han sido cuestionados hasta la fecha aunque, como se podrá comprobar, el número asignado a las epístolas en las ediciones difiere en muchos casos de su colocación original en el manuscrito ${ }^{6}$. En este artículo trataremos de analizar los contenidos y la disposición de estas epístolas en el citado manuscrito León 22, así como sus relaciones con algunas de las otras cartas para apuntar posibles soluciones a los problemas creados por la curiosa ordenación anticronológica de estos elementos en el Epistularium de Braulio de Zaragoza.

de Sevilla, A, B, I-VI (1-8 según la numeración que les impuso Risco), que preceden a las Etymologiae del Hispalense, se citarán según la edición de Lindsay 1911.

${ }^{5}$ Este valioso códice ha recibido la atención de numerosos estudiosos y existe gran cantidad de bibliografía sobre él; la puesta al día más reciente la realiza Martín 2006, pp. 104-105.

${ }^{6}$ Por este motivo se proporcionará, junto con el número identificativo de la epístola, que se mantiene en todas las ediciones del Epistularium completo, la referencia a los folios que esa epístola ocupa en León, Archivo Capitular, 22. 
1. Estado de la cuestión. La disposición de las epístolas de Braulio de Zaragoza en el manuscrito León, Archivo Capitular, 22

En primer lugar, debemos advertir que el manuscrito presenta un problema en los folios donde están copiadas estas epístolas. En el f. 75ra se interrumpe el texto de la epístola 32 y se inserta el fragmento final de una epístola que Tajón dirige a Braulio, en la que le consulta algunas cuestiones sobre la autenticidad de las reliquias (Riesco 1975, p. 154). Sigue la respuesta de Braulio a esta misiva, un pequeño tratado sobre los atributos de los cuerpos gloriosos $^{7}$ (epístola 42, Riesco 1975, pp. 154-162). Tras esta, encontramos una epístola consolatoria dirigida a un personaje llamado Nebridio, con motivo de la muerte de su esposa (Riesco 1975, pp. 136-138). Sin embargo, esto no afecta a la sucesión de las epístolas de las que nos ocupamos. En el f. 75ra encontramos el siguiente aviso: hic minus abet per errantia scriptoris, sed in quinta folia pergameni, ubi signam inueneris, recte legebis. Similitudo hec signe Ø. Si eliminamos la interpolación de las dos epístolas y el fragmento, la sucesión es tal y como se ha descrito más arriba, sin que haya ningún tipo de omisión en los textos ni duda sobre su organización ${ }^{8}$. Como se puede deducir de la tabla que se presenta a continuación, la inserción de elementos procedentes de otras partes de Epistularium pero ajenos a este subconjunto se produce en la cuarta pieza de la sucesión, la 32: es obvio que 32 sigue a 36 , aunque su texto se interrumpa, y el orden lógico de 3132-33 es, a pesar de este problema, incontestable. Por tanto, este «error del copista» no afecta al estudio de la disposición de estas cinco epístolas, ya que se hace evidente que se han copiado en un orden preciso y que su particular sucesión no ha sido motivada por el problema textual que acabamos de mencionar; creemos, pues, poder analizar la organización de estas epístolas sin tenerlo directamente en cuenta. Presentamos a continuación un esquema

7 Sobre estas epístolas, sus circunstancias históricas y las relaciones entre los dos religiosos, Orlandis 2003, pp. 403-407 (el capítulo dedicado a Tajón está incluido también en Orlandis 1992, pp. 128-143)

${ }^{8}$ Sobre este problema, su posible resolución y las diversas hipótesis sobre sus causas, Lynch 1950, pp. 239-240, propone una omisión voluntaria de determinados fragmentos por parte de Tajón, que él supone compilador y organizador del Epistolario de Braulio, hipótesis no demostrable científicamente y que no se apoya en datos objetivos. En cambio, Gil 1971, p. 141, aventura el desplazamiento de un cuaternión como causa para este problema, lo que parece encajar con la extensión y naturaleza de los textos transpuestos. 
detallado de los contenidos de este grupo de epístolas y de su disposición en el ms. 22 de León y en las ediciones del Epistularium de Braulio, su incipit y una referencia a sus contenidos:

Tabla 1

\begin{tabular}{|c|c|c|c|}
\hline \multicolumn{2}{|r|}{ Ms. 22} & \multirow{2}{*}{$\begin{array}{c}\text { Eds. } \\
35\end{array}$} & \multirow{2}{*}{$\begin{array}{l}\text { Año } \\
646 / 7\end{array}$} \\
\hline f. $71 r-72 r$ & $\begin{array}{l}\text { EUGENIO A BRAULIO.- - incipit: Duae res obortae sunt } \\
\text { (consulta sobre un problema en la ordenación de un presbítero) }\end{array}$ & & \\
\hline f. $72 \mathrm{r}-74 \mathrm{r}$ & $\begin{array}{l}\text { BRAULIO A EUGENIO-- incipit: Si inmensa curarum genera } \\
\text { (respuesta a la consulta) }\end{array}$ & 36 & $646 / 7$ \\
\hline f. $74 r-75 r$ & $\begin{array}{l}\text { BRAULIO a CHINDASVINTO.- incipit: Dominus omnipotens } \\
\text { (petición de que permita a Eugenio permanecer en Zaragoza) }\end{array}$ & 31 & $645 / 6$ \\
\hline f. $75 \mathrm{r}$ & $\begin{array}{l}\text { CHINDASVINTO A BRAULIO.- incipit: Suggessionem eloquentie vestre } \\
\text { (reiteración de la orden por la cual Eugenio debía trasladarse a Toledo } \\
\text { para ser consagrado obispo) }\end{array}$ & & \\
\hline f. $75 \mathrm{r}-79 \mathrm{r}$ & $\begin{array}{l}\text { (interrupción del texto-inserción de las epistolas 34, Frag. y 42)- } \\
\text { aviso de la interrupción del texto y su localización }\end{array}$ & & \\
\hline f. $79 r-79 v$ & (epístola 32-continuación) & 32 & $645 / 6$ \\
\hline f. $79 v$ & $\begin{array}{l}\text { BRAULIO a ChINDASVINTO.- incipit: Etsi disrupto potius quam scisso } \\
\text { (aceptación de la orden del rey y envío de Eugenio a Toledo) }\end{array}$ & 33 & $645 / 6$ \\
\hline
\end{tabular}

Este grupo de epístolas se divide, como se puede apreciar en la tabla anterior, en dos subgrupos. Las epístolas 35 y 36 datan de los primeros tiempos del episcopado de Eugenio, esto es, a partir del 646, ya que describen una situación que se le presentó al obispo recién llegado y que remontaba a los tiempos de su predecesor ${ }^{9}$. Eugenio había encontrado irregularidades en la ordenación de un presbítero de su sede y escribió a su maestro, solicitando consejo sobre cómo actuar. Braulio responde a la consulta mediante el recurso a las Escrituras y a la literatura patrística; podemos suponer que esta contestación se envió casi inmediatamente, por la gravedad del asunto tratado (con consecuencias teológicas y pastorales de gran importancia) y por los

9 Sobre los títulos de tratamiento que Braulio y Eugenio utilizan en estas epístolas y su interpretación, Sánchez Salor 1964, pp. 30-31. 
ruegos de Eugenio, que en varias ocasiones apremia a Braulio a que le proporcione una solución urgente:

Quid inde fieri praecipit uestra prudentia, cita me iussione certifica ... Inde per eum te precor, cuius dono beatus, cuius instructione peritus esse probaris et doctus, ut me de his causis celerrime sacra tui oris iussione certifices. (Braul., Epist. 35, 1. 15; Alberto 2005, p. 399, 11. 39-40, ibíd., p. 400).

El siguiente grupo de tres epístolas (dos enviadas por Braulio al rey Chindasvinto y la respuesta de este) deben datarse forzosamente en el período inmediatamente anterior a la elevación episcopal de Eugenio, esto es, en el 645/646. Braulio, ya anciano, no puede prescindir de la ayuda de su arcediano Eugenio para sus labores pastorales y, tras recibir seguramente una orden de Chindasvinto, que no se nos ha conservado, expresando sus deseos de consagrar a Eugenio obispo de Toledo, contesta intentando que el rey cambie de opinión para conservar a su lado a su querido discípulo. El monarca no cede e insiste, en la epístola 32, en que Eugenio se presente en Toledo para su consagración episcopal. En la epístola 33 Braulio, aunque entristecido por la separación de su amigo, accede a las órdenes reales y envía a Eugenio a la corte toledana.

Se hace obvio de este pequeño resumen de los argumentos que este grupo de tres epístolas, 31-33, debe preceder a 35-36; esta ruptura extremadamente violenta del orden de envío de la correspondencia es única en el Epistularium. Una cosa es que un grupo de piezas epistolares no respete una sucesión cronológica cuando no existen indicaciones que permitan datarlas, como sucede en gran parte de la correspondencia de Braulio de Zaragoza, y otra muy diferente es una alteración del orden lógico que se puede percibir mediante una simple lectura de las epístolas. Este problema es difícilmente explicable como un accidente de la tradición manuscrita, ya que absolutamente todos los demás grupos de epístolas que suponen el desarrollo lógico y cronológico de un argumento se hallan en el epistolario dispuestas en perfecto orden. Véanse, por ejemplo, el grupo de cuatro epístolas intercambiadas con Recesvinto sobre la corrección del Forum Iudicum (Miguel Franco 2006), dispuestas en orden cronológico y respetando lo que debió de ser la alternancia del intercambio epistolar: Braulio, en la epístola 38, pide disculpas por la tardanza en el envío de un códice cuya corrección le había sido encargada. En la 39 el rey Recesvinto se muestra comprensivo con las dificultades de Braulio y le anima a que continúe con su labor. La epístola siguiente (40) acompañó sin duda al envío 
del códice ya corregido por el obispo; en la última de la serie (41), Recesvinto agradece a Braulio su esfuerzo y alaba los resultados de su corrección (Riesco 1975, pp. 152-154). Sirve como ejemplo asimismo la sucesión de consultarespuesta en las epístolas 43 y 44 (Riesco 1975, pp. 162-182), que son respectivamente una serie de cuestiones de exégesis bíblica que Fructuoso de Braga propone a Braulio y la respuesta de este último.

Por este motivo, el primer editor del Epistularium, M. Risco (1775, pp. 363-373), dispuso estas epístolas en el orden cronológico en el que han sido editadas hasta nuestros días, comentando los motivos para esta ordenación en el prólogo antepuesto a su edición (Risco 1775, sin paginación). Madoz 1945, p. 44, en su estudio de la cronología y la disposición de las piezas del Epistularium, sigue principalmente la autoridad de Risco en lo que respecta a la datación de las piezas y su consiguiente reorganización dentro del conjunto, de acuerdo con la progresión lógica de la sucesión del intercambio epistolar. Para estas epístolas, que estaban «enteramente dislocadas» en el manuscrito, se impone la «recta ordenación» que llevó a cabo el anterior editor (Madoz 1945, pp. 52-53). L. Riesco conserva también este orden tradicional y considera la disposición irregular de estas cinco epístolas una prueba de que la ordenación cronológica que se aprecia en el Epistularium no es ni mucho menos rigurosa (Riesco 1975, pp. 10-11).

Sin embargo, la resolución del problema que plantea la disposición de estas epístolas va más allá de su reorganización por parte del editor para recrear el orden en el que debieron de ser enviadas. Este es el único caso en el que el Epistularium, en el manuscrito 22 de León, presenta una alteración de este tipo en el orden cronológico de sus piezas, por lo que se hace necesario tratar de encontrar un motivo para esta particular organización antes de descartarla en las ediciones como un mero error. Para ello, se puede tomar como punto de partida el análisis del orden general de las piezas de este Epistularium y, en concreto, de los contenidos de estas epístolas, así como la comparación de los significados creados por esta curiosa sucesión dentro del contexto del Epistularium completo.

\section{El ORDEN DEL EPISTVLARIVM: LA ESTRUCTURA BIMEMBRE}

Antes de comenzar con nuestra argumentación, debemos puntualizar que, aunque las epístolas que Braulio e Isidoro intercambiaron sobre la redacción de las Etymologiae y su envío a Braulio se han editado juntas, como si forma- 
sen parte de una sola colección epistolar, es probable que en origen no fuesen un conjunto unitario. El primer grupo, de epístolas isidorianas, que fue añadido por Braulio a su recensión de las Etymologiae (Codoñer 2005, pp. 274299); como ya hemos dicho, este primer grupo se encuentra entre los ff. 38va44rb de León 22, además de en una gran mayoría de los ejemplares de las Etymologiae que han llegado hasta nosotros. El resto de la correspondencia del obispo de Cesaraugusta está contenido entre los ff. 51ra y 88vb. Entre los dos conjuntos se insertan otras obras de Braulio: en los folios 44ra-48va encontramos las actas del proceso entre los obispos Marciano y Habencio y, entre los folios 48va y 51ra, la Professio fidei (CPL, núm. 1233) propuesta a los judíos toledanos. Por otra parte, ambos grupos, el conjunto isidoriano y el de la correspondencia general, parecen tener una entidad propia, basada en características compartidas por todas las piezas que los componen, sin que en principio se puedan englobar en una superestructura común. Nos centraremos, por tanto, en este segundo grupo de epístolas, dejando de lado las ocho primeras intercambiadas con Isidoro (Miguel Franco 2010).

Los escrúpulos que surgen al analizar el orden de las epístolas según las encontramos en León 22 derivan principalmente de lo poco que sabemos sobre este manuscrito y sobre su modelo de copia: ¿quién dispuso así las epístolas? ¿Podemos hacer remontar este orden a Braulio o se debe a un mero accidente de la transmisión? Paradójicamente, la disposición de las obras en este valioso testimonio manuscrito es demasiado caprichosa para deberse al sentido común del copista y tiene demasiada coherencia para deberse al azar.

En general, los editores han coincidido en que las piezas que componen el Epistularium brauliano están organizadas en un esquema bimembre. Madoz afirma que, aunque no se aprecia una distribución lógica en la colocación de las epístolas, el epistolario parece sin embargo estar dividido en dos mitades: la primera está ocupada por cartas familiares y consolatorias; la segunda, por cartas de negocios y doctrinales (Madoz 1945, p. 33). Pero se podrían realizar ulteriores matizaciones a esta apreciación de Madoz. La frontera entre las dos mitades la marca una de las epístolas más importantes del conjunto: la carta que Braulio redacta en el 638 en nombre del VI Concilio de Toledo para refutar las acusaciones del papa Honorio I, que había reprochado a la Iglesia hispana su poco rigor hacia los judíos y judaizantes ${ }^{10}$, y que recibe el núme-

10 Fita realizó una edición de esta epístola (1870, pp. 261-277), en la que se incluye más documentación de la época y un estudio detallado: 1870, pp. 187-204, pp. 260-278; 1871, 
ro 21 en las ediciones (Riesco 1975, pp. 108-114). Esta posición de la epístola 21 responde a las convenciones tradicionales sobre la estructura de las obras compuestas de elementos individuales, como los poemarios o los epistolarios: el inicio, el centro y el final son los lugares marcados, reservados a las piezas más relevantes o que por algún motivo se quieren destacar.

Pero la organización de las epístolas no se limita a la división en dos grupos de extensión semejante en torno a una pieza central que sobresale por importancia y posición. Existen otros elementos que proporcionan a este Epistularium una gran cohesión interna a la vez que construyen la particular progresión narrativa: la ligazón entre las piezas se consigue incluyéndolas en una estructura que las engloba y las sitúa de modo que se creen relaciones entre ellas. En la segunda parte del Epistularium, a la citada epístola 21 a Honorio I (ff. 65rb-67vb) suceden las epístolas 25-27 (ff. 67vb-69va) de Braulio y el abad Emiliano de Toledo (García Moreno 1974, núm. 90; Kampers 1979, núm. 131), que fue valido de Chindasvinto y gozó de gran poder y prestigio en la capital toledana. Ambos religiosos intercambian delicadezas y palabras de admiración y afecto; además, el de Cesaraugusta solicita el envío del tratado sobre el Apocalipsis de Apringio de Beja, que Emiliano dice no haber podido encontrar. Tras estas, se insertan tres epístolas consolatorias a diversos destinatarios: en el f. 69va-b la epístola 28 (Riesco 1975, p. 126) a Ataúlfo (García Moreno 1974, núm. 54), en los ff. 69vb-70va la epístola 29 (Riesco 1975, p. 128) dirigida a Gundesvinda y Givario (García Moreno 1974, núm. 37) y, finalmente, en los ff. 70va-71rb la epístola 30 (Riesco 1975, p. 130) a Wistremiro (García Moreno 1974, núm. 102). A continuación, en los ff. 71ra-74rb encontramos las citadas epístolas 35 y 36, de Eugenio y Braulio.

Varios puntos parecen importantes de esta sucesión. En primer lugar, los destinatarios religiosos (el papa Honorio I, el abad Emiliano, el obispo Eugenio) están agrupados $\mathrm{y}$, en especial, destaca que se presenten seguidas las epístolas a destinatarios religiosos relacionados con Toledo, como Emiliano y Eugenio. En segundo lugar, las epístolas consolatorias están dispuestas de modo que separen misivas de características diferentes, como las de Emiliano, que podrían formar parte de la correspondencia cotidiana y en las que no se trata ningún asunto especial, y el bloque de correspondencia del que nos

pp. 271-279, pp. $358-365$, pp. 447-58; 1871, pp. 49-60, pp. 101-107, pp. 192-200; pp. 252260, pp. 336-346, pp. 403-29. Aproximaciones recientes a este texto, García Moreno 2005, pp. 104-107, Martín 2006, p. 39. 
ocupamos, cuyo tema reviste gran importancia y que tienen que ver con la relación de Braulio con la monarquía. Estas tres epístolas consolatorias quedarían directamente enfrentadas, considerando la estructura bimembre del Epistularium, a otras tres (18-20) que ocupan el mismo lugar en la primera parte, esto es, contando a partir de la epístola central a Honorio, tendrían el cuarto, quinto y sexto puesto. Parecen tener la misma función: se intercalan entre grupos de misivas dirigidas a destinatarios religiosos, una de ellas, la de Wiligildo, que trata, como veremos más adelante, un tema de importancia relativo a la ordenación de un diácono, y las cartas a Eutropio y Valentín, en las que se discuten asuntos de índole intelectual y personal.

Tabla 2

\begin{tabular}{|l|l|}
\hline \multirow{2}{*}{ epist. 17: Wiligildo (ff. 58vb-59va) } & epist. 36: Eugenio (f. 72ra-74rb) \\
\cline { 2 - 2 } & epist. 35: Eugenio a Braulio (f. 71ra-72ra) \\
\hline epist. 18: Pomponia (ff. 59va-60va) & epist. 30: Wistremiro (f. 70va-71rb) \\
\hline epist. 19: Hoyón y Eutrocia (ff. 60va-62ra) & epist. 29: Gundesvinda y Givario (f. 69vb-70va) \\
\hline epist. 20: Hoyón y Eutrocia (f. 62ra-62va) & epist. 28: Ataulfo (f. 69va-69vb) \\
\hline epist. 22: Eutropio (ff. 62vb-63vb) & epist. 27: Emiliano (f. 69ra-69va) \\
\hline epist. 23: Valentín (ff. 63vb-64vb) & epist. 26: Emiliano a Braulio (f. 68va-69ra) \\
\hline epist. 24: Valentín (ff. 64vb-65rb) & epist. 25: Emiliano (f. 67vb-68va) \\
\hline \multicolumn{2}{|l}{ epist. 21: Honorio (ff. 65rb-67vb) } \\
\hline
\end{tabular}

Del mismo modo, a las epístolas 31-33 (ff. 74rb-75ra; f. 79va ${ }^{11}$ ) suceden la 37 (f. 79vb-80rb), dirigida a Chindasvinto, y las mencionadas 38-41 (ff. 80va-81vb), intercambiadas con Recesvinto. Las epístolas a destinatarios religiosos quedan agrupadas y, a continuación, las misivas que Braulio intercambió con los reyes forman también un grupo homogéneo.

Esta disposición de las epístolas responde a un patrón preciso; el cuidado que se aprecia en la estructura de la colección y en la agrupación de los elementos no puede sino haber sido motivado por un deseo de construir una obra cerrada y significativa en su conjunto, así como un intento de subrayar determinadas características de algunas piezas.

11 V. las consideraciones realizadas más arriba sobre la interrupción de la copia de esta epístola. 
III. CRONOLOGÍA Y NARRACiÓN EN LAS EPÍSTOLAS 31 A 36 del EpistVlarivm de Braulio de Zaragoza

\section{Creación de las imágenes de Braulio y de Eugenio}

Como hemos apuntado más arriba, en esta segunda parte se concentran las epístolas que Madoz definió epístolas de negocios, en las que Braulio trata temas relacionados con las tareas administrativas de su episcopado. En esta época Braulio, como obispo de Cesaraugusta y gracias a su prestigio intelectual, estaba en una posición desde la que podía intervenir en asuntos legislativos y políticos ${ }^{12}$. En la epístola 32, entre las razones que el rey Chindasvinto aduce para reclamar la presencia de Eugenio en Toledo leemos:

Nam maximum exinde ante Dominum consequi poterit premium, dum speculatorem eum relaxaueris immolandum, tuaque sublimior aput $\dagger$ diuinam $\dagger$ effici poterit laudatio, si ex tuis doctrinis sancta catholica prefulserit eclesia (Braul., Epist. 32, 11. 22-26; Riesco 1975, p. 134) ${ }^{13}$.

Tenemos, en primer lugar, una petición al rey denegada, lo que no habla bien de la influencia de Braulio sobre el monarca. Si la función de muchos epistolarios es la de resaltar determinados aspectos de la personalidad de su autor, como han subrayado a lo largo de los años los estudiosos de las colecciones epistolares (Schmeidler 1949, pp. 220-238, Constable 1976, p. 58), estas cartas a Chindasvinto podrían no haber sido incluidas, ya que hablan de un rey que hace caso omiso a las sugerencias del autor del Epistularium. Sin embargo, la frase anterior justifica enteramente no sólo la inclusión de las tres epístolas intercambiadas con Chindasvinto, sino también da un sentido nuevo a las epístolas 35 y 36.

En una lectura de las epístolas en la sucesión en la que nos las encontramos en el manuscrito de León, el lector se encuentra primero con la consulta de Eugenio a Braulio, que, por el tono en el que se realiza, coloca a nuestro autor en una posición de superioridad moral e intelectual. Tras esta exposi-

12 Sobre la conjunción entre los aspectos del poder real y del poder episcopal que se produjo durante los reinados de Chindasvinto y Recesvinto, v. Collins 2004, pp. 83-89.

${ }_{13}$ Para resolver el problema textual marcado, †diuinam†, Gil 1971, p. 145, propone la adición de un sustantivo, como gloriam, clementiam, iustitiam. Riesco en su edición opta por añadir pietatem. 
ción de los méritos de Braulio, encontramos la correspondencia con el rey, en la que Braulio se autocalifica de anciano, enfermo e incapaz de cumplir con las obligaciones del episcopado y menciona en modo emotivo el cariño que le une a Eugenio. El rey, tras negar que el de Cesaraugusta esté perdiendo facultades, a juzgar por el contenido y elaboración retórica de su epístola, reclama nuevamente a Eugenio, asegurándole que la fama del maestro es un motivo para la elección del discípulo y que el apoyo a Eugenio redundará en mayor gloria de Braulio. La presentación de estos elementos se hace de modo que parezca que esta exaltación de la figura de Braulio se realiza «a su pesar»: él parece interesado solamente en la continuación de su labor pastoral, preocupado por sus achaques e inseguro por lo delicado de la consulta de Eugenio, mientras que Eugenio y Chindasvinto apelan a su sabiduría y valía y actúan en consecuencia con la alta opinión que tienen de Braulio.

En el pasaje que acabamos de citar, el rey asegura a Braulio que su gloria será mayor si su sabiduría viene en apoyo de la Iglesia Católica: esta afirmación tiene carácter universal, se refiere al conjunto de la Iglesia por la expresión y no sólo a la Iglesia de Zaragoza, como se deduce del sintagma sancta catholica ... ecclesia. Es decir, los méritos de Braulio aumentarán no tanto si él es capaz de dirigir sin la ayuda de Eugenio la diócesis zaragozana, sino, muy especialmente, si su doctrina se añade a aquellas que constituyen los cimientos sobre los que se sustenta el conjunto de los católicos. Esta interpretación se apoya también en las palabras de Eugenio en la Epist. 35, 11. 2-3: duae res obortae sunt in ecclesia tua, unde nimium contabescit anima mea (Alberto 2005, p. 399). Mediante el adjetivo posesivo tua se subraya la responsabilidad de Braulio sobre la Iglesia de Toledo y su ascendente sobre Eugenio $^{14}$.

La legitimación de la intervención de Braulio en los asuntos de la Iglesia de Toledo es doble: se justifica por la petición del propio Eugenio y por la frase del rey, que, al hacer derivar los méritos de Eugenio de la doctrina de su maestro Braulio, coloca al de Cesaraugusta en una posición de responsabilidad en lo que respecta a la Iglesia de Toledo. No podemos saber si esta

${ }^{14}$ Risco introduce aquí la corrección mea, llevado por el sentido inmediato de la referencia a la Iglesia de Toledo por parte de Eugenio, que fue aceptada también por Madoz. Alberto conserva en su edición este posesivo de segunda persona (tua), lo que parece conveniente por el contexto general, ya que Eugenio recalca de este modo la responsabilidad de Braulio en la iglesia toledana. 
afirmación se debe a una confianza real en las capacidades y la fama de Braulio o es una mera excusa para evitar el enfrentamiento con él.

De este modo, las epístolas 35 y 36 adquieren un nuevo sentido: en ellas se concreta la sublimior ... laudatio de Braulio. La brusca ruptura del orden temporal de las epístolas puede ser en este caso un poderoso mecanismo narrativo, que permite comenzar in medias res la narración de la historia de la elevación episcopal de Eugenio y el papel que en ella tuvieron Braulio y Chindasvinto, así como la influencia de Braulio sobre el rey y sobre Eugenio, para después proporcionar los elementos que permiten darle sentido al conjunto.

\section{La imagen de Chindasvinto en las epistolas: referencias intratextuales e intertextuales}

Como apuntábamos más arriba, la construcción de un esquema que cohesione las piezas de una obra de este tipo, como puede ser un epistolario o un poemario, suele basarse tanto en la disposición de los elementos como en la creación de relaciones entre ellos; estos elementos trascienden así su condición individual para funcionar en pares o grupos. De este modo, no sólo se refuerza la caracterización de la obra como estructura cerrada y perfecta en sí misma más allá de su constitución a partir de elementos individuales, sino que las piezas adquieren nuevos matices y significados mediante la comparación, superposición y contraste con otras.

En las obras de estructura bimembre es frecuente que este tipo de correspondencias se produzcan entre piezas paralelas, es decir, la estructura se refuerza con una disposición especular que enfrente pares o grupos de piezas de modo que la combinación o contraste de sus contenidos subraye alguno de sus aspectos. Un poderoso mecanismo de cohesión entre elementos es la reiteración del léxico $y$, en menor medida, la utilización de intertextos o fuentes semejantes.

La alteración de la sucesión cronológica puede responder también a la intención de llamar la atención sobre las particularidades de la relación de Braulio con Eugenio, por una parte, y con el poder real en este período, por otra. No se puede olvidar que a la petición fallida de Braulio a Chindasvinto sigue otra, la epístola 37 (Riesco 1975, p. 148), en la que se pide a Chindasvinto que asocie a la monarquía a su hijo Recesvinto, que sí surtió efecto. 
Tomando como punto de partida la estructura bimembre del Epistularium, las epístolas 31, 32 y 33, situadas en la segunda parte, se corresponderían con la epístola 17 de la primera parte, que ocupa el noveno lugar de la sucesión en el manuscrito y está situada más o menos en el centro de la primera parte; contando a partir de la pieza central, la número 21 , que sería la decimosexta en el Epistularium de León 22, las epístolas 31 a 33 ocuparían los puestos noveno a undécimo, estando situadas asimismo en el centro del segundo bloque de cartas. La equivalencia se apoya también en la recurrencia del léxico y la semejanza de los contenidos. El análisis de esta epístola, como veremos más adelante, nos puede dar algunas de las claves para la interpretación de la correspondencia de Braulio con Chindasvinto.

La epístola 17 a Wigildo es la disculpa de Braulio a un obispo, cuya sede no ha sido identificada ${ }^{15}$, por haber ordenado diácono sin su autorización expresa a un monje de su diócesis (Martin 2007, pp. 215-216). Esto iba en contra de los cánones de numerosos concilios ${ }^{16}$. Si comparamos las epístolas 33 a 36 con esta epístola 17, que ocupa la novena posición de la primera parte, vemos que tratan del mismo tema: de la ordenación de un religioso en contra de la voluntad de su superior. En un caso, es Braulio el que ordena un presbítero sin consultar con el obispo del que depende; en el otro, es Chindasvinto el que dispone la consagración episcopal de Eugenio haciendo caso omiso de las súplicas del obispo Braulio. En el primer caso, Braulio pide disculpas y afirma que actuó guiado por el amor que une a todos los cristianos y que está por encima de cualquier otra regla, incluidas las que derivan de las jerarquías eclesiásticas; también en el segundo grupo apela al vínculo del cariño que le une con Eugenio.

15 Madoz, 1945 p. 48; en p. 115 propone identificar a Ayulfo, mencionado en esta carta, con Aviulfo de Aquitania, que interviene en un caso análogo, el de un clérigo que había huido a Hispania. Wiligildo podría ser identificado con Wilsigisilo, obispo de Toulouse más o menos en la misma época (627), lo que explicaría el comentario de Braulio sobre la distancia de las respectivas sedes episcopales. Sin embargo, esta identificación no pasa de ser una hipótesis.

${ }^{16}$ Como, por ejemplo, los cánones 15 a 17 del I Concilio de Nicea (Alberigo 2007, pp. 177-178). Esto se recoge en numerosos concilios, anteriores y contemporáneos a Braulio (e. g., Reg. Eccl. Carthag. 80, Vt de alieno monasterio susceptos nec praepositos monasterii nec clericos liceat ordinare, Munier 1974, p. 204, 1. 734). Braulio dice haber cumplido los requisitos pertinentes para la ordenación, esto es, la comprobación de la virtud del ordenando (que se repiten en los cánones de diversos concilios, como Conc., Asp. c. 5, De Clercq 1963, p. 164). 
En el plano léxico, se aprecia en todas estas epístolas la recurrencia del término ordinatio y del verbo ordinare, tanto con el significado específico de 'consagrar a un religioso' como en sentido más general, aunque siempre dentro del contexto eclesiástico, que hace referencia al tema común de los textos. Estos términos no se encuentran con este sentido más que en estas dos piezas de la correspondencia de Braulio.

Nam cum haec ipsa recogito quod, quamuis diuersitate rectorum in multis partiatur ordinatio ecclesiarum (Braul., Epist. 17, 11. 12-13; Riesco 1975, p. 98).

Et ideo in hunc famulum uestrum ordinationem dedi (Braul., Epist. 17, 11. 18-19; Riesco, íbíd.).

Ille autem, qui arcana et secreta perlustrat et necessitates nostras pensitat, animis uestris inspiret qualiter sic unam eclesiam ordinetis, ut aliam non destituatis (Braul., Epist. 31, 11. 31-33; Riesco 1975, p. 132)

cedat necesse est quod mortalis uolumtas expetit adque, dum gloriae uestrae ordinatio suppleta fuerit, commendamus quanta possumus obsecratione eius deplorandam peregrinationem (Braul., Epist. 33, 11. 10-12: Riesco 1975, p. 136)

Del mismo modo, encontramos los verbos sacrare y consacrare:

cum monacum uestrum de asili monasterio ... et subdiaconem et diaconem sacrasse (Braul., Epist. 17, 11. 5-6; Riesco 1975, p. 98)

quod ipse hic extiterit oriundus, ubi nunc eum consecrandum speculatorem obtamus (Braul., Epist. 32, 11. 18-19; Riesco 1975, p. 134)

Se hace obvio de esta comparación que la disposición enfrentada de estas epístolas fue planeada cuidadosamente por Braulio; el sutil juego de alusiones a un tema que podría ser delicado, por tratarse del cumplimiento de una orden del monarca, deja entrever que Braulio, aun habiendo obedecido, no había olvidado el asunto, ni se había conformado con la negativa de Chindasvinto, ya que procuró subrayar que la ordenación episcopal de Eugenio se había realizado en contra de la voluntad del superior que debía autorizarla. 
3. El enfrentamiento con el poder y la posición de Braulio: mecanismos de expresión

La utilización de fuentes semejantes o las referencias intertextuales a deteminados autores pueden funcionar como mecanismo de cohesión entre diversas piezas. Una de las fuentes identificadas de la epístola 33 parece confirmar esta interpretación. Se trata de las Enarrationes in psalmos, de Agustín de Hipona, obra que Braulio conoce y utiliza a menudo:

Etsi disrupto potius quam scisso societatis uinculo, quo cum seruo tuo Eugenio arcediacono in Domino conectebar (Braul., Epist. 33, 11. 4-5; Risco 1975, p. 136).

et sic accipiunt 'in uanitate ciuitates suas', id est, populos suos uanos, eorum uanitatem sectantes; cum inflati iustitiae nomine persuadent ut disrupto unitatis uinculo, eos tamquam iustiores caeci et imperiti sequantur (Aug., In psalm. 138.26, 11. 16-19; Dekkers-Fraipont 1956, p. 2008).

Si aceptamos que Braulio no tomó estas palabras por su simple belleza formal, sino por su adecuación al contexto, vemos que podríamos además relacionarlas con las afirmaciones del rey en la carta precedente:

Nec enim, sub hac uestra postulatione, nostra est praetermittenda iustitia, quod ipse hic extiterit oriundus, ubi nunc eum consecrandum speculatorem optamus.

Ergo, quia nostrae parti procul dubio patet iustitia, nostrae deuotioni quae promissa sunt non fraudabitur (Braul., Epist. 32, 11. 16-21; Riesco 1975, p. 134).

Se deduce de la frecuente utilización de esta obra agustinana que Braulio la conocía bien; que se contaba entre los fondos de la biblioteca de Cesaraugusta es obvio por una petición de Isidoro en la epístola B, que precede a las Etymologiae, en la que el Hispalense solicita: Dum pariter essemus, postulaui te ut mihi decadam sextam sancti Agustini transmitteres (Lindsay 1911, p. 3). La expresión disrupto uinculo no es frecuente en la literatura latina; el contexto de donde Braulio sin duda lo tomó es además coherente con el contexto donde lo inserta. Se aprecia también la reiteración de otros términos significativos como iustitia en la carta a Chindasvinto (nostrae parti procul dubio 
patet iustitia) y en el texto agustiniano (inflati iustitiae nomine persuadent...). Es, con toda probabilidad, un reproche a la actuación del rey al llevarse a Eugenio a Toledo, reforzado además por los paralelismos establecidos por la posición de la epístola 17. Braulio deja ver más de las epístolas de lo que las epístolas mismas nos dicen, mediante alusiones solapadas que, en este caso en concreto, no habría podido manifestar explícitamente, por respeto tanto al rey como al ministerio de Eugenio.

Se ha sugerido ya que las palabras de Ildefonso de Toledo en su De uiris illustribus dejan entrever cierta hostilidad hacia el rey: unde principali uiolentia reductus atque in pontificatum adscitus (Ildeph., Vir. 13, 11. 5-6; Codoñer 2007, pp. 485-616), aunque podría igualmente tratarse de un tópico que subraya la humildad de Eugenio ya que sólo a la fuerza y en contra de su voluntad aceptó el cargo de obispo y los honores que conllevaba (Alberto 2005, pp. 13-14, n. 5) ${ }^{17}$. Sin embargo, combinadas con el contenido de las epístolas y otras informaciones insertadas de diversos modos en el Epistularium, las palabras de Ildefonso de Toledo podrían recoger no un tópico, sino un enfrentamiento real de Chindasvinto no con Eugenio, sino con Braulio.

Como se ha dicho anteriormente, las cartas de Eugenio y Braulio están próximas a las que Emiliano y Braulio intercambiaron. Emiliano, importante personaje estrechamente relacionado con el poder real, responde de la siguiente manera a Braulio, que había solicitado le enviase el tratado sobre el Apocalipsis de Apringio de Beja:

Pro libro autem quem inquisitone mea inuentum dirigendum bestre beatitudini precepistis, testis est mihi Deus quia omni intentione quesiui ... sed omnino iste codex inueniri non potuit, nam et pro libris Laurenti solliciti fuimus, sed, quia illo tempore res, sicut nostis, in dispersionem uenit, nihil inde inuestigare potuimus (Braul., Epist. 26, 11. 10-16; Riesco 1975, p. 124).

Cuando Chindasvinto alcanzó el trono, llevó a cabo diversos actos de represión contra sus opositores; la dispersión de los libros del conde Loren-

${ }^{17}$ Los cánones de diversos concilios habían decretado explícitamente la invalidez de la ordenación episcopal de un religioso en contra de su voluntad, como Conc., Aurel. 11: Item, sicut antiqui canones decreuerunt, nullus inuitis detur episcopus, sed nec per oppressionem pontentium personarum ad consensum faciendum ciues aut clirici ... inclinentur. Quod si factum fuerit, ipse episcopus, qui magis per uiolentiam quam per decretum legitimum ordinatur, ab indepto pontificatur honore in perpetuo deponatur (De Clercq 1963, p. 152). 
zo es consecuencia de la caída en desgracia de este personaje al comienzo del reinado de Chindasvinto (García Moreno 1976, núm. 90). La expresión sicut nostis es una alusión velada a los problemas políticos y sociales que se desencadenaron al comienzo del reinado. Las epístolas a Emiliano sirven de introducción al enfrentamiento que más tarde tendría lugar entre Braulio y el monarca, presentando el contexto del reinado de Chindasvinto y algunos de los acontecimientos negativos que trajo consigo.

De este modo se nos ofrece una descripción no sólo de las relaciones de Braulio con Chindasvinto, sino de la opinión que Braulio y otros intelectuales de la época tuvieron sobre la figura del monarca y algunas de sus actuaciones. A pesar de que Braulio mantenía una relación bastante cordial con la corte toledana, sus ideas sobre determinados asuntos no coincidían con las de Chindasvinto (Castellanos 2004, pp. 291-300, v. especialmente p. 295): la imagen del rey que Braulio crea mediante la disposición de las epístolas y sus contenidos es ilustrativa de los mecanismos de los que la intelectualidad visigoda se valió para expresar sus opiniones y su descontento con el poder.

\section{Conclusiones}

Un análisis cuidadoso del orden del Epistularium de Braulio de Zaragoza puede proporcionar datos importantes no sólo sobre la estructura de la obra, sino sobre las intenciones de Braulio y sus opiniones acerca de los hechos expuestos en sus epístolas. Este estudio ha de realizarse sin aplicar ideas apriorísiticas sobre el orden deseable o esperable en un conjunto de epístolas, sino observando simplemente los datos que poseemos, tanto cronológicos como codicológicos y combinándolos con el análisis léxico, de fuentes y narratológico de las epístolas. De este modo, aparece ante nuestros ojos no sólo la cuidada arquitectura de la antología epistolar brauliana, sino también el juego de significados creado por esta disposición. Estos significados pueden tener gran relevancia para el conocimiento de la historia del periodo; de este modo, la particular disposición de las epístolas que Braulio intercambió con Chindasvinto nos informa del enfrentamiento entre el poder real y el obispo de Cesaraugusta debido a lo que este sintió como una intrusión en el terreno de exclusividad del poder episcopal, esto es, la ordenación de un presbítero de su diócesis, con el que además mantenía una relación de amistad. Así, Braulio crea una imagen de Chindasvinto y expone su opinión sobre lo sucedido de un modo que, a pesar de su sutileza, no deja lugar a dudas. Se pone 
asimismo de manifiesto la relación de afecto y admiración intelectual entre Braulio y Eugenio, es más, se potencia por encima de la difícil relación con el monarca mediante un mecanismo simple y efectivo, como es la inversión del orden lógico de la correspondencia. En resumen, Braulio, utiliza lo que en esta época hemos dado en llamar ready made: conjuga realia (es decir, epístolas reales) con mecanismos artístico-literarios para crear una obra en la que se combinan de modo efectivo verdad histórica, narración personal y expresión de la subjetividad.

\section{BIBLIOGRAFÍA}

Alberigo, G. 2007: Conciliorum Oecumenicorum Generaliumque Decreta, editio critica, I. The oecumenical councils from Nicaea I to Nicaea II (325-787), Turnhout, Brepols (CC COGD 1).

Alberto, P. F. 2005: Eugenii Toletani Episcopi Opera Omnia, Turnhout, Brepols (CC SL 114).

Castellanos, S. 2004: La hagiografia visigoda. Dominio social y proyección cultural, Logroño, Universidad de la Rioja.

Codoñer C. 2005: «Etymologiae», en Chiesa, P. y Castaldi, L. (eds.), La trasmissione dei testi latini del Medioevo. Mediaeval Latin Texts and their transmission, Te.Tra. II, Florencia, Sismel, pp. 274-299.

Codoñer, C. 2007: «Ildefonsi Toletani Episcopi. De uiris illustribus», en Yarza Urkiola, V. y Codoñer, C. (eds.), Ildefonsi Toletani Episcopi De Virginitate Sanctae Mariae. De cognitione Baptismi. De itinere Deserti, De uiris illustribus, Turnhout, Brepols (CC SL 114A), pp. 485-616.

Collins, R. 2004: Visigothic Spain 409-711, Oxford, Blackwell.

Constable, G. 1976: Letters and letter collections, Turhnout, Brepols (Typologie des sources du Moyen Age occidental 17).

CPL: Dekkers, E. y Gaar, A. 1995: Clavis Patrum Latinorum, Turnhout, Brepols.

De Clercq, Ch. 1963: Concilia Galliae, A. 511-697, Turnhout, Brepols (CC SL 148A).

Dekkers, E. y Fraipont, J. 1956: Sancti Aurelii Augustini Enarrationes in Psalmos CI-CL, Turnhout, Brepols (CC SL 40).

Fita, F. 1870-1871: «El Papa Honorio I y San Braulio de Zaragoza», La Ciudad de Dios 1, 4 (1870), pp. 187-204, pp. 260-278; 2, 5 (1871), pp. 271-279, pp. 358$365,447-458$; 2, 6 (1871), pp. 49-60, pp. 101-107, pp. 192-200, pp. 252-260, pp. 336-346, pp. 403-429.

García Moreno, L. A. 1974: Prosopografia del reino visigótico de Toledo, Salamanca, Universidad de Salamanca. 
García Moreno, L. A. 2005: Los judios de la España Antigua, Madrid, Rialp.

Gil, J. 1971: «Sobre el texto de las cartas de S. Braulio», Cuadernos de Filología Clásica 2, pp. 141-146.

Kampers, G. 1979: Personengeschichtliche Studien zum Westgotenreich in Spanien, Múnich, Aschendorffsche.

Lindsay, W. M. 1911: Etymologiae siue Originum libri XX, Oxford, Clarendon Press.

Lynch, C. H. 1950: San Braulio de Zaragoza (631-651). Su vida y sus obras, Madrid, Instituto Enrique Flórez (obra editada, anotada y traducida por P. Galindo).

Madoz, J. 1945: Epistolario de S. Braulio de Zaragoza. Edición crítica según el códice 22 del Archivo Capitular de León, con una introducción histórica y comentario, Madrid, CSIC.

Martín, J. C. 2006: Scripta de uita Isidori Hispalensis episcopi, Turnhout, Brepols (CC SL 113B).

Martin, C. 2007: «Les évêques visigothiques dans leur espace: de l'autonomie à l'intégration», en Bougard, F., Depreux, Ph. y Le Jan, R. (eds.), Les élites et leur espace: mobilité, rayonnement, domination (VIe - XIe s.), Turnhout, Brepols, pp. 207-223.

Miguel Franco, R. 2006: «Braulio de Zaragoza y la corrección del Fuero Juzgo», Helmantica 57, pp. 67-89.

Miguel Franco, R. 2010: «El orden del Epistularium de Braulio de Zaragoza», en Bautista Pérez, F. y Gamba Corradine, J. (eds.), Estudios sobre la Edad Media, el Renacimiento y la temprana Modernidad, San Millán de la Cogolla, Semyr, pp. 289-299.

Munier, Ch. 1963: Concilia Galliae A. 314-A. 506, Turnhout, Brepols (CC SL 148).

Munier, Ch. 1974: Concilia Africae, A. 345-A. 525, Turnhout, Brepols (CC SL 149).

Orlandis, J. 1992: Semblanzas visigodas, Madrid, Rialp.

Orlandis, J. 2003: Historia del Reino visigodo español: los acontecimientos, las instituciones, la sociedad, los protagonistas, Madrid, Rialp.

Riesco, L. 1975: Epistolario de Braulio de Zaragoza. Introducción, edición crítica y traducción, Sevilla, Universidad de Sevilla.

Risco, M. 1775: «Epistulae Braulionis», en Flórez, E. y Risco, M. (eds.), España Sagrada, vol. 30, Madrid, pp. 318-395.

Sánchez Salor, E. 1964: Jerarquías eclesiásticas y monacales en la época visigótica, Salamanca, Universidad de Salamanca.

Schmeidler, B. 1949: «Die Briefsammlung Froumunds von Tegernsee», Historisches Jahrbuch 62-69, pp. 220-238. 
BRAULIO DE ZARAGOZA, EL REY CHINDASVINTO Y EUGENIO DE TOLEDO 175

Valcárcel, V. 1991: «Sobre el origen geográfico de la familia de Braulio, obispo de Zaragoza», en Ramos Guerreira, A. (ed.), Mnemosynum C. Codoñer a discipulis oblatum, Salamanca, Universidad de Salamanca, pp. 333-340.

Fecha de recepción de la primera versión del artículo: 30/04/2010

Fecha de recepción de la versión definitiva del artículo: 24/11/2010

Fecha de aceptación: 19/11/2010 